\title{
Edge Enhancing Accelerated Diffusion Model for Speckle Denoising in Medical Imagery
}

\author{
Arundhati Bagchi Misra ${ }^{1, *}$, Chartese Jones ${ }^{2, * *}$, and Hyeona $\operatorname{Lim}^{2, * * *}$ \\ ${ }^{1}$ Saginaw Valley State University, USA \\ ${ }^{2}$ Mississippi State University, USA
}

\begin{abstract}
Speckle noise occurs in a wide range of medical images due to sampling and digital degradation. Removing speckle noise from medical images is the key for further automated processing techniques like segmentation, and can help the clinicians with better diagnosis and therapy. We consider partial differential equation (PDE)-based denoising model which is a modified EulerLagrange equation derived from the total variation minimization functional with additional speckle noise constraints. The new PDE model is designed and optimized to rectify speckle noise and enhance edges present in medical imagery. We also develop the efficient and stable discretization techniques for the corresponding speckle denoising model. The method is tested for several types of images including ultrasound images, and it is compared favorably to the conventional denoising model.
\end{abstract}

\section{Introduction}

Images occur in many forms in our daily life and denoising is one of the most important steps in image analysis to improve the quality of images. It is also used as a preprocessing for other imaging techniques such as feature segmentation and compression. Various image denoising methods have been developed for the last two to three decades and one of the most fundamental techniques is using the partial differential equations (PDEs) from energy minimization [1]-[9]. In 1992, Rudin et al. [3] proposed a variational technique using the total variation (TV) minimization for effective denoising. Kim and Lim [9] further improved the TV scheme by developing a non-convex minimizing functional for simultaneous denoising and edge enhancement. However, these methods are mainly for the images corrupted by the Gaussian noise which is simple and additive. That is, they assumed that an observed (noisy) image $f$, contaminated by the Gaussian noise $n$, is represented by the equation

$$
f=u+n,
$$

where $u$ is the original noise free image. Therefore, these models do not work very well on the images with more complicated noise such as speckle noise. One of the most widely used images having speckle noise is ultrasound image. Ultrasound provides low cost images that are extremely helpful for clinical diagnosis. However, the ultrasound process results in

\footnotetext{
*e-mail: abmisra@svsu.edu

**e-mail: cdj192@msstate.edu

***e-mail: hlim@math.msstate.edu
} 
images corrupted with specks of artifacts. Currently, medical professionals are trained to derive meaningful information from these images. But automatic processing is not possible because of the noise and artifacts present in the image. Therefore, before using any of these images, it is very important to reduce the effect of speckle noise present in them. Speckle noise is granular in nature and it exists inherently in the image. Unlike Gaussian noise, which affects single pixels of an image, speckle noise affects multiple pixels. Hence it is not possible to remove the speckle noise well with the traditional Gaussian denoising models. Several speckle denoising models and their algorithms have been developed to tackle these types of images [10]-[14]. Among them, Krissian et al. [10] developed a new restoration scheme as a energy minimization constrained by the special noise equation. Their method is based on the total variation (TV) minimization scheme for Gaussian noise developed by Rudin et al. [3]. Their scheme can effectively remove speckle noise present in ultrasound images. The method was further improved by Lim and Williams [12] by introducing a nonstandard PDE based anisotropic diffusion model for efficient edge-preserving denoising for speckle noise images.

In this paper, a new model and its stable algorithm for speckle denoising based on the model by Krissian et al. [10] is introduced. The new model is inspired by the realistic speckle noise equation in [12] and it will also have the edge enhancing feature by incorporating the non-convexity of the functional introduced by [9]. In Section 2, we further discuss more details on the conventional speckle denoising model by Krissian et al. [10]. In Section 3 , a new model for speckle denoising is introduced. Numerical procedure and stability of the new model is also discussed in this section. The numerical results in Section 4 show that the new model outperforms the Krissian et al. model. Finally, we summarize our results in Section 5 .

\section{Preliminary}

Krissian et al. [10] assumed the speckle noise to be multiplicative. The noise equation was experimentally obtained and was given by

$$
f=u+\sqrt{u} n
$$

instead of Eq. (1), which is for the images contaminated by the Gaussian noise. Since $n=\frac{f-u}{\sqrt{u}}$, the following TV based minimization functional is obtained:

$$
\min _{u} F(u)=\min _{u}\left(\int_{\Omega}\left[|\nabla u|+\frac{\lambda}{2}\left(\frac{f-u}{\sqrt{u}}\right)^{2}\right] d \mathbf{x}\right) .
$$

Here, $\mathbf{x} \in \Omega$ and $\lambda$ is a constraint parameter. From energy minimization of this functional and after adding artificial time marching, the Krissian et al.'s TV-based speckle denoising model was derived as:

$$
\frac{\partial u}{\partial t}-\frac{u^{2}}{f+u} \nabla \cdot\left(\frac{\nabla u}{|\nabla u|}\right)=\lambda(f-u) .
$$

This model can effectively remove speckle noise compared to other conventional TV Gaussian denoising models. However, it was later noticed by Lim and Williams [12] that the coefficient of the diffusion term $\nabla \cdot\left(\frac{\nabla u}{|\nabla u|}\right)$ is $\frac{u^{2}}{f+u}$, which can be approximated as $u / 2$ assuming that $f \approx u$. This coefficient makes the diffusion faster in the lighter region and slower 
in the darker region, which is unrealistic. Thus the authors in [12] proposed the following noise equation

$$
f=u+\left(\sqrt{u-f_{s}}\right) n,
$$

where $f_{s}$ denotes the smoothed version of the noisy image $f$. It was studied in [12] that the term $u-f_{s}$ in Eq. (5) induces the selective diffusion in different regions so that the method removes the noise more effectively.

\section{Edge Enhancing Accelerated Diffusion (EEAD) model}

\subsection{The new model}

According to our observation, the speckle model by Krissian et al. [10] is quite efficient and the model by Lim and Williams [12] is more realistic. Keeping this in mind, we developed a new model incorporating the positive aspects of both. Here, we propose the following form of a noise equation:

$$
f=u+\left(\sqrt{u+C\left|u-f_{s}\right|^{\alpha}}\right) n
$$

which gives

$$
n=\frac{f-u}{\sqrt{u+C\left|u-f_{s}\right|^{\alpha}}}
$$

Kim and Lim in 2007 [9] developed a non-convex minimizing functional and a corresponding model for Gaussian denoising. It has been shown that their model gives more accurate denoising results than the conventional TV models and it has edge enhancing features. By combining the idea by [9] with Eq. (7), we develop the general regularized functional minimization problem given by

$$
\min _{u} F(u)=\min _{u}\left(\int_{\Omega}\left[|\nabla u|^{p}+\frac{\lambda}{2}\left(\frac{f-u}{\sqrt{u+C\left|u-f_{s}\right|^{\alpha}}}\right)^{2}\right] d \mathbf{x}\right) .
$$

By introducing the energy descent direction by an artificial time $t$ and by adopting the strategy developed by Marquina and Osher [6] to prevent the staircasing effect of the TV based models, the equivalent Euler-Lagrange equation to Eq. (8) is modified to the following nonlinear PDE:

$$
\frac{\partial u}{\partial t}=\left(u+C\left|u-f_{s}\right|^{\alpha}\right)|\nabla u|^{2-p} \nabla \cdot\left(\frac{\nabla u}{|\nabla u|^{2-p}}\right)+\lambda|\nabla u|^{2-p}(f-u), 0<p<1 .
$$

We call this equation the Edge Enhancing Accelerated Diffusion (EEAD) model. The EEAD model will be numerically solved iteratively with no-flux boundary condition and with the given noisy image $f$ as an initial data. The numerical solution to the EEAD model (9) will still approximate the solution $u$ to the minimizing functional (8).

\subsection{Numerical procedure}

Following the linearized time stepping procedure introduced by Cha and Kim in [15], we develop the explicit numerical scheme for our EEAD model (9):

$$
\begin{aligned}
u_{i j}^{n+1}=u_{i j}^{n}+M_{i j}^{n} \Delta t\left[A_{W} u_{i+1, j}^{n}+A_{E} u_{i-1, j}^{n}+\right. & \left.A_{N} u_{i, j+1}^{n}+A_{S} u_{i, j-1}^{n}-4 u_{i j}^{n}\right] \\
& +\beta_{i j}^{n} \Delta t\left(f_{i j}-u_{i j}^{n}\right)
\end{aligned}
$$


where

$$
\begin{gathered}
A_{W}=\frac{2 d_{i j, W}}{d_{i j, W}+d_{i j, E}}, A_{E}=\frac{2 d_{i j, E}}{d_{i j, W}+d_{i j, E}}, \\
A_{N}=\frac{2 d_{i j, N}}{d_{i j, N}+d_{i j, S}}, A_{S}=\frac{2 d_{i j, S}}{d_{i j, N}+d_{i j, S}}, \\
M_{i j}:=u_{i j}^{n}+C\left|u_{i j}^{n}-f_{s, i j}\right|^{\alpha},
\end{gathered}
$$

and $\beta_{i j}$ is the central difference approximation of $\lambda|\nabla u|^{2-p}$ at $\left(x_{i}, y_{j}\right)$ pixel. Here, $|\nabla u|$ is

replaced by $\left|\nabla^{\epsilon} u\right|:=\sqrt{u_{x}^{2}+u_{y}^{2}+\epsilon^{2}}$, where $\epsilon \ll 1$ is the regularization parameter. This is to prevent the denominator $|\nabla u|^{2-p}$ in the EEAD model (9) from approaching zero. $d_{i j, W}$ is the approximation of $\left|\nabla^{\epsilon} u\right|^{2-p}$ at $\left(x_{i-1 / 2}, y_{j}\right)$ pixel. For approximating $u_{y}$ at $\left(x_{i-1 / 2}, y_{j}\right)$, central difference and standard averaging of two adjacent values are used. $d_{i j, E}, d_{i j, N}, d_{i j, S}$ are defined similarly.

\subsection{Numerical stability}

The following theorem analyzes the stability of the numerical scheme in Eqs. (10)-(11) adapted for the EEAD model (9).

Theorem 3.1 Define $b^{\star}=\max _{i, j, n}\left(u_{i j}^{n}+C\left|u_{i j}^{n}-u_{s, i j}\right|^{\alpha}\right), \beta=\max _{i, j, n} \beta_{i, j}^{n}$ and let

$$
\left(4 b^{\star}+\beta\right) \Delta t \leq 1
$$

Then the numerical scheme in Eqs. (10)-(11) is stable.

Proof. It is sufficient to show the following inequality:

$$
\min _{i, j} f_{i j} \leq u_{i j}^{n} \leq\|f\|_{\infty}
$$

By (12), $\left(4 M_{i j}^{n}+\beta_{i j}^{n}\right) \Delta t \leq\left(4 b^{\star}+\beta\right) \Delta t \leq 1$. Let $u_{i j}^{n+1}$ be a local minimum. Then

$$
\begin{aligned}
u_{i j}^{n+1} & \geq u_{i j}^{n}+\Delta t M_{i j}^{n}\left(A_{W}+A_{E}+A_{N}+A_{S}\right) u_{i j}^{n+1}-4 \Delta t M_{i j}^{n} u_{i j}^{n}+\beta_{i j}^{n} \Delta t\left(f_{i j}-u_{i j}^{n}\right) \\
& =\left[1-\left(4 M_{i j}^{n}+\beta_{i j}^{n}\right) \Delta t\right] u_{i j}^{n}+4 \Delta t M_{i j}^{n} u_{i j}^{n+1}+\beta_{i j}^{n} \Delta t f_{i j} \\
& \geq\left[1-\left(4 M_{i j}^{n}+\beta_{i j}^{n}\right) \Delta t\right] u_{i j}^{n+1}+4 \Delta t M_{i j}^{n} u_{i j}^{n+1}+\beta_{i j}^{n} \Delta t f_{i j}, \\
& =u_{i j}^{n+1}-\beta_{i j}^{n} \Delta t u_{i j}^{n+1}+\beta_{i j}^{n} \Delta t f_{i j} .
\end{aligned}
$$

This implies that $u_{i j}^{n} \geq f_{i j}$, for all $i, j$. Similarly, the second inequality of (13) can be proved by setting $u_{i j}^{n+1}$ to be a local maximum. The inequality (13) is now proved and the scheme in Eqs. (10)-(11) is stable.

\section{Numerical Results}

In this section, we test our algorithm developed in Section 3.2 for the EEAD model and compare the results with the Krissian et al. model. For this purpose, we use four different images: 'Block' (cartoon), 'Lenna', and human 'Gallstone' and 'Liver' ultrasound images. Block, Lenna, and Liver images are of size $256 \times 256$ and Gallstone is of $322 \times 290$. The summary of numerical results are given in Table 1, Figure 1, Figure 2, and Figure 3. 
Table 1. Comparison table for computation time and PSNR using Krissian et al. and EEAD applied to Block, Lenna, and ultrasound images (Gallstone and Liver).

\begin{tabular}{|c||c|c||c|c|}
\hline \multicolumn{1}{|c||}{} & \multicolumn{2}{c||}{ Krissian et al. } & \multicolumn{2}{c|}{ EEAD } \\
\cline { 2 - 5 } & Time (s) & PSNR & Time (s) & PSNR \\
\hline \hline Block (PSNR=28.03) & 2.85 & 30.05 & 3.68 & 32.57 \\
Lenna (PSNR=25.70) & 2.88 & 28.23 & 3.63 & 30.01 \\
Gallstone & 4.06 & - & 5.19 & - \\
Liver & 2.83 & - & 3.64 & - \\
\hline
\end{tabular}

(a)

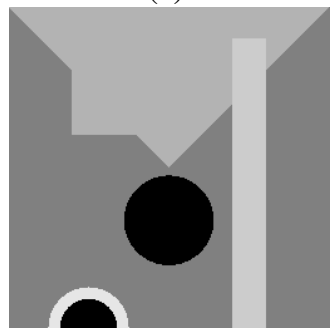

(b)

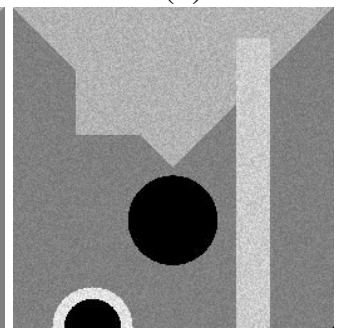

(c)

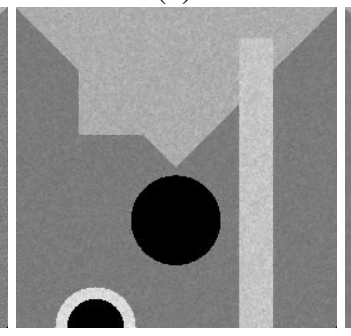

(d)

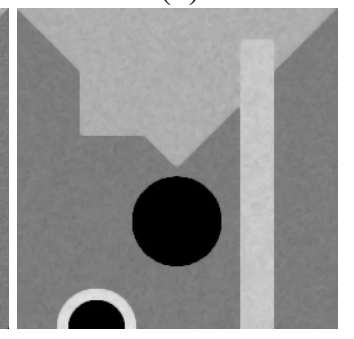

Figure 1. Block: (a) Original, (b) Speckle Noisy (with PSNR=28.03), (c) Krissian et al., and (d) EEAD.

(a)

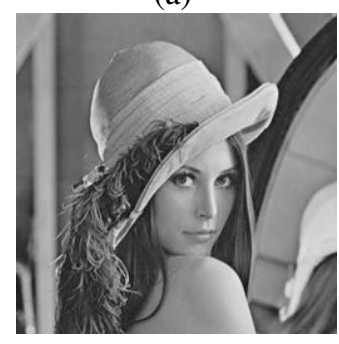

(b)

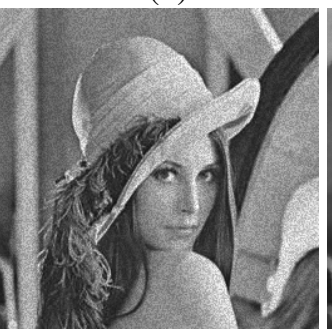

(c)

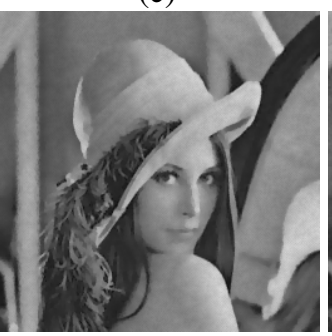

(d)

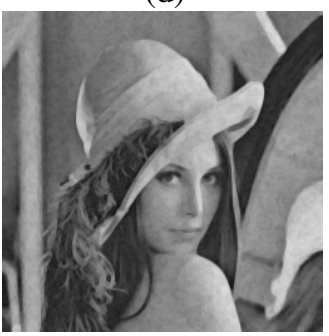

Figure 2. Lenna: (a) Original, (b) Speckle Noisy (with PSNR=25.70), (c) Krissian et al., and (d) EEAD.

The peak-signal-to-noise ratio (PSNR) is defined as follows to measure the accuracy of the methods:

$$
\text { PSNR }=10 \log _{10}\left(\frac{\sum_{i j} 255^{2}}{\sum_{i j}\left(g_{i j}-u_{i j}\right)^{2}}\right) .
$$

Here, $g$ is the noise free clean image and $u$ is the restored image obtained by denoising a noisy version of $g$.

For all four images, the non-convex parameter $p$ was set to 0.5 when using the EEAD model. For Block and Lenna, we chose $\Delta t=0.001$ and $\epsilon=0.05$ for both models. We also set $C=10, \alpha=0.02, \beta=50$ for EEAD and $\lambda=15$ for Krissian et al. For ultrasound images, $\epsilon$ was chosen as 0.005 for EEAD and 0.001 for Krissian et al. We set $C=2, \alpha=0.02, \beta=200$ for EEAD and $\lambda=3$ for Krissian et al. 
(a)

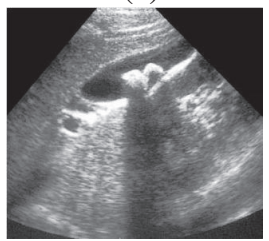

(b)

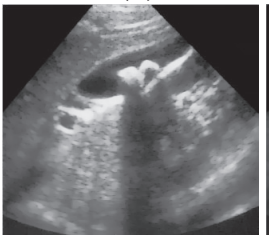

(c)

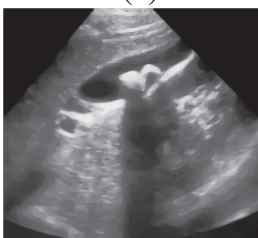

(d)

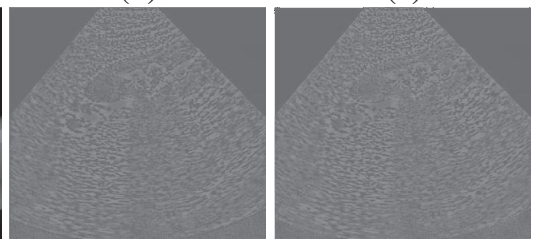

Figure 3. Gallstone: (a) Original, (b) Krissian et al., (c) EEAD, and speckle noise residuals for (d) Krissian et al. and (e) EEAD.

(a)

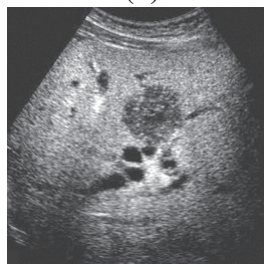

(b)

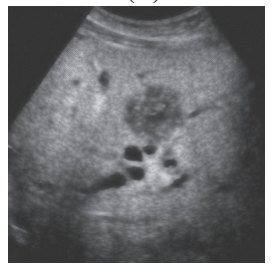

(c)

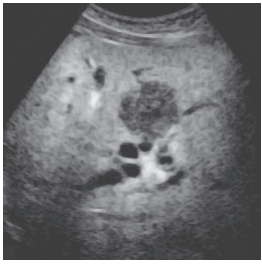

(d)

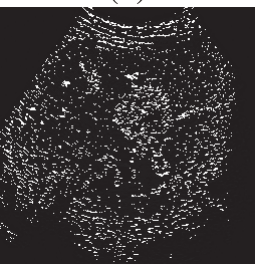

(e)

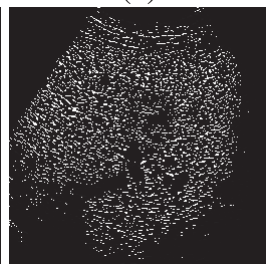

Figure 4. Liver: (a) Original, (b) Krissian et al., (c) EEAD, and speckle noise residuals for (d) Krissian et al. and (e) EEAD.

Table 1 provides execution time for all four images and PSNR values were measured on Block and Lenna when speckle noise was synthetically added. For ultrasound images: Gallstone and Liver, denoising models were directly applied to them without adding any noise artificially since the images themselves contain natural speckle noise. Therefore, the PSNR are not applicable for these two images. As one can see from Table 1, computation time was slightly increased for the EEAD model. This is expected since the coefficient $\left(u+C\left|u-f_{s}\right|^{\alpha}\right)$ of the diffusion term in EEAD is more complicated than the one in Krissian et al. The PSNR results in Table 1 and visual verification in Figure 1 and Figure 2 indicate that the EEAD produces more accurate results than the Krissian et al. model for images with synthetically added noise. It can also be seen in Figure 3 and Figure 4 that the EEAD performs better than the Krissian et al. for ultrasound images with natural speckle noise. For a closer inspection, we calculated the speckle noise residual $(n=(f-u) / \sqrt{u})$ for Gallstone and Liver, and included the results in Figure 3 and Figure 4. Here, the intensities for the residual images were flipped and enhanced for a better recognition of noise. The residuals of the Krissian et al. model contains a lot more unnecessary details from the images which could result in the loss of important features of the images. The EEAD model mainly picked up the noise while keeping the overall structure of the images. It can also be clearly seen in the figure that the edges of denoised images using EEAD are sharper than the ones using the Krissian et al. and this is due to the non-convexity of the new model. This result implies that EEAD is better on detecting possible locations of abnormality and therefore more useful in clinical diagnosis than the Krissian et al. model.

\section{Conclusions}

In this article, the authors have developed more accurate speckle denoising model and its stable numerical methods. The model is based on the TV minimization and the associated non-linear PDE, and Krissian et al.'s speckle noise equation model. The method is more useful in medical imagery especially for ultrasound images since the ultrasound process results 
in images with complex types of noise and unclear edges. Realistic and efficient speckle noise equation model was introduced and edge enhancing feature by adopting non-convexity of the functional was added to the new model. An effective numerical scheme was introduced and its stability was proved. Several numerical examples show that the new model outperforms the Krissian et al. on their accuracy. The method was also tested on ultrasound images with natural artifacts and specks. Possible locations of abnormalities were better identified after reducing the natural noise using the new model.

\section{References}

[1] D. Mumford and J. Shah, "Optimal approximations by piecewise smooth functions and variational problems," Commun. Pure Appl. Math., vol. 42, pp. 577-685,(1989)

[2] P. Perona and J. Malik, "Scale-space and edge detection using anisotropic diffusion," IEEE Trans. Pattern Anal. Mach. Intell., vol. 12, no. 7, pp. 629-639, (1990)

[3] L. I. Rudin, S. Osher, and E. Fatemi, "Nonlinear total variation based noise removal algorithms," Physica D, vol. 60, pp. 259-268, (1992)

[4] F. Catté, P.-L. Lions, J.-M. Morel, and T. Coll, "Image selective smoothing and edge detection by nonlinear diffusion," SIAM J. Numer. Anal., vol. 29, no. 1, pp. 182-193, (1992)

[5] L. Alvarez, P.-L. Lions, and J.-M. Morel, "Image selective smoothing and edge detection by nonlinear diffusion. ii," SIAM J. Numer. Anal., vol. 29, no. 3, pp. 845-866, (1992)

[6] A. Marquina and S. Osher, "Explicit algorithms for a new time dependent model based on level set motion for nonlinear deblurring and noise removal," SIAM J. Sci. Comput, vol. 22, pp. 387-405, (1999)

[7] T. F. Chan, G. H. Golub, and P. Mulet, "A nonlinear primal-dual method for total variation-based image restoration," SIAM J. Sci. Comput., vol. 20, pp. 1964-1977, (1999)

[8] A. Chambolle, "An algorithm for total variation minimization and applications," J. Math. Imaging Vision, pp. 89-97, (2004)

[9] S. Kim and H. Lim, "A non-convex diffusion model for simultaneous image denoising and edge enhancement," Electro. J. of Diff. Eq., vol. 15, pp. 175-192, (2007)

[10] K. Krissian, R. Kikinis, C.-F. Westin, and K. Vosburgh, "Speckle-constrained filtering of ultrasound images," in Proc. IEEE Computer Society Conf. Computer Vision and Pattern Recognition, Sand Diego, CA, Jun. 2005, vol. 2, pp. 547-552

[11] K. Krissian, C.-F. Westin, R. Kikinis, and K. Vosburgh, "Oriented Speckle Reducing Anisotropic Diffusion," ” IEEE Trans. Image Process., vol. 16, no. 5, pp. 1412-1424, (2007)

[12] H. Lim and T. N. Williams, "A non-standard anisotropic diffusion for speckle noise removal," Journal of Systemics, Cybernetics and Informatics, vol. 5, no. 2, pp. 12-17, (2007)

[13] A. B. Misra and H. Lim, "Nonlocal speckle denoising model based on non-linear partial differential equations," Information Systems Design and Intelligent Applications, pp 165176, (2015)

[14] A. B. Misra, E. Lockhart, and H. Lim, “Total variation based denoising methods for speckle noise images," Involve, a J. of Mathematics, vol. 10, no. 2, pp 327-344, (2016)

[15] Y. Cha and S. Kim, "Edge-forming methods for image zooming," J. Math. Imaging Vision, vol. 25 pp. 353-364 (2006) 